\title{
Stratifications on the Ran Space
}

\author{
Jānis Lazovskis ${ }^{1}$ (iD
}

Received: 20 December 2019 / Accepted: 28 April 2021 / Published online: 11 June 2021

(C) The Author(s) 2021

\begin{abstract}
We describe a partial order on finite simplicial complexes. This partial order provides a poset stratification of the product of the Ran space of a metric space and the nonnegative real numbers, through the Čech simplicial complex. We show that paths in this product space respecting its stratification induce simplicial maps between the endpoints of the path.
\end{abstract}

Keywords Stratifications $\cdot$ Simplicial complexes $\cdot$ Configuration space $\cdot$ Entrance path

\section{Introduction}

The Ran space of a topological space $X$ is, as a set, all finite subsets of $X$, endowed with a topology to allow points on $X$ to split and nearby points to merge [6]. The Ran space decomposes into configuration spaces, where the size of the subset of $X$ does not change. Configuration spaces have been applied [12] to study the topology of the space $X$, and the Ran space has been used $[3,13]$ to make vast generalizations about $\infty$-categories.

Stratifications were originally [20] meant to generalize smooth manifolds, but have since become $[13,19]$ a broader tool to decompose topological spaces with respect to a poset. Often stratifications are required to be conical [4], meaning every point has a neighborhood that looks like a stratified cone. The Ran space has a natural stratification into its constituent configuration spaces, and we are interested in refining this by not only considering the number of points in a subset of $X$, but also the distance among the points.

This approach takes us directly to persistent homology [11], which combines a topological perspective at a range of distances. The topological features that are present for longer are considered essential to the topology of the underlying space, a perspective that has proven to be useful in a wide range of applications $[2,16,17]$. The most common product of persistent homology is the persistence diagram, which provides a clean way to convey observations [21]. Topological spaces based on persistent homology have been studied [10], with a notion of distance coming from comparing such persistence diagrams.

This paper is based on the doctoral thesis of Jānis Lazovskis, defended May 2019 at the University of Illinois at Chicago.

Jānis Lazovskis

janis.lazovskis@abdn.ac.uk

1 Institute of Mathematics, University of Aberdeen, Aberdeen AB24 3UE, Scotland, UK 


\subsection{Motivation}

Persistent homology uses filtrations of algebraic objects [7], most often simplicial complexes, to produce persistence diagrams. Simplicial complexes have been used $[1,14]$ to probe the topology of the underlying space. A key aim of this exposition is to combine the filtration of a particular simplicial complex with the different choices of simplicial complexes that can be made by sampling a space. With such a combination, we are motivated to answer the following questions:

- If there is a path in from one finite sample of $M$ to another:

- can the changes in filtrations between these samples be precisely described?

- can the persistent homology computation of a new sample be simplified by using the results of a different sample?

- Can we construct a space of all possible persistence diagrams by keeping track of homological changes of the simplicial complex coming from a sample of $M$ and a distance $r$, as both change?

These questions, some of which have been already considered [5], would be greatly helped along if $\operatorname{Ran}(M) \times \mathbf{R} \geqslant 0$ were stratified. The first component in the product is the choice of a finite sample of $M$, and the second component is the persistent homology distance parameter.

A stratification needs a poset, so we define a novel partial order on isomorphism classes of simplicial complexes in Definition 5. This poset [SC] prompts in own questions:

- What are the order relations on this poset?

- Is the order complex of this poset shellable?

- Are there subposets of [SC] that are not shellable?

- Does there exist an oriented coloring on [SC]?

This is the first introduction, to our knowledge, of this poset.

\subsection{Overview}

The scope of the present work is to stratify $\operatorname{Ran}(M) \times \mathbf{R}_{\geqslant 0}$. Fix $M$ to be a metric space, and to a finite subset $P \subseteq M$, build the Čech complex. This simplicial complex has $P^{\prime} \subseteq P$ defining a $\left(\left|P^{\prime}\right|-1\right)$-simplex whenever the intersection of (closed) $r$-balls around the points of $P^{\prime}$ is non-empty. We denote by $\breve{C}$ this assignment of a simplicial complex to a pair $(P, r) \in \operatorname{Ran}(M) \times \mathbf{R}_{\geqslant 0}$ of a sample $P$ of $M$ and a nonnegative distance $r$.

Our contributions are first in the introduction of a partial order $\succcurlyeq$ on isomorphism classes of simplicial complexes [SC] in Section 3.2. We also introduce the concept of a "frontier simplicial complex" that refines the notion of a simplicial complex in Section 4.1. The results on [SC] extend to results on [FSC], the poset of isomorphism clasases of frontier simplicial complexes.

Using the poset $[\mathrm{SC}]$ as a stratifying set, we prove our main results:

- (Theorem 1) $\operatorname{Ran}(M) \times \mathbf{R} \geqslant 0$ is [SC]-stratified

- (Theorem 2) $\operatorname{Ran}(M) \times \mathbf{R}_{\geqslant 0}$ is conically stratified if $M$ is semialgebraic

These results are interpreted in the context of out persistent homology motivation to relate the filtrations of different samples of $M$. In Section 4.2 we observe that the Čech filtration 
arising of a finite subset of $M$ is always subposet of [SC]. Finally, we show in Construction 1 that every path $\gamma$ in $\operatorname{Ran}(M) \times \mathbf{R} \geqslant 0$ that respects its stratification induces a unique simplicial $\operatorname{map} \check{C}(\gamma(0)) \rightarrow \check{C}(\gamma(1))$.

\section{Background}

Let $\mathrm{SC}$ be the set of finite, abstract simplicial complexes. ${ }^{1}$ A simplicial complex $C$ is defined by its vertices and simplices, that is, a pair of sets $(V(C), S(C))$ with $S(C) \subseteq P(V(C))$ closed under taking subsets.

\subsection{Topological Spaces}

Let $X$ be a topological space. The Ran space of $X$ is $\operatorname{Ran}(X):=\{P \subseteq X: 0<|P|<\infty\}$, with topology defined as the coarsest ${ }^{2}$ for which all maps $X^{I} \rightarrow \operatorname{Ran}(X)$ are continuous for every nonempty finite set $I$ [6, Section 3.4.1]. This condition may be equivalently stated in terms of images of open sets through them map $X^{I} \rightarrow \operatorname{Ran}(X)$ [13, Definition 5.5.1.2], and once a metric has been chosen on $X$, is equivalent to the Hausdorff distance on subsets of $X$ [13, Remark 5.5.1.5].

Let $M$ be a metric space with metric $d$. For a positive integer $n$, write $\operatorname{Conf}_{n}(M)$ and $\operatorname{Ran}_{\leqslant n}(M)$ for the subspaces of $\operatorname{Ran}(M)$ with elements exactly of size $n$ and at most size $n$, respectively. In the former case, $\operatorname{Conf}_{n}(M)$ is called the unordered configuration space of $n$ points.

Recall the Hausdorff distance between $P, Q \in \operatorname{Ran}(M)$ is defined as

$$
\begin{aligned}
d_{H}(P, Q) & :=\max \left\{\max _{p \in P} \min _{q \in Q} d(p, q), \max _{q \in Q} \min _{p \in P} d(p, q)\right\} \\
& =\min \left\{r: Q \subseteq \bigcup_{p \in P} B(p, r), P \subseteq \bigcup_{q \in Q} B(q, r)\right\} .
\end{aligned}
$$

We write $B$ for the open ball in $M$ and $\bar{B}$ for the closed ball in $M$. The Hausdorff distance is an upper bound for a coarser metric $d_{M}$ on $M$, as

$$
d_{M}(X, Y):=\inf _{x \in X, y \in Y}\{d(x, y)\} \leqslant d_{H}(X, Y),
$$

for any $X, Y \subseteq M$. On the product space $\operatorname{Ran}(M) \times \mathbf{R}_{\geqslant 0}$ we use the sup-norm

$$
d_{\infty}((P, r),(Q, s)):=\max \left\{d_{H}(P, Q),|r-s|\right\} .
$$

Definition 1 Given a pair $(P, r) \in \operatorname{Ran}(M) \times \mathbf{R}_{\geqslant 0}$, the $\check{C}$ ech complex on $P$ with radius $r$ is the simplicial complex with vertices $P$, and $P^{\prime} \subseteq P$ a simplex whenever $\bigcap_{p \in P^{\prime}} \bar{B}(p, r) \neq$ $\emptyset$. This assignment $\check{C}: \operatorname{Ran}(M) \times \mathbf{R}_{\geqslant 0} \rightarrow \mathrm{SC}$ is called the $\check{C} e c h$ map.

Some of the spaces we are interested in are semialgebraic. Recall that a set in $\mathbf{R}^{N}$ is semialgebraic if it can be expressed as a finite union of sets of the form

$$
\left\{x \in \mathbf{R}^{N}: f_{1}(x)=0, \ldots, f_{m_{1}}(x)=0, g_{1}(x)>0, \ldots, g_{m_{2}}(x)>0\right\},
$$

\footnotetext{
${ }^{1}$ All simplicial complexes will be abstract and finite unless otherwise noted, so we drop the adjectives.

${ }^{2}$ Used in the sense that all other topologies satisfying the condition contain at least the same open sets as the given topology.
} 
for polynomial functions $f_{i}, g_{i}: \mathbf{R}^{N} \rightarrow \mathbf{R}$.

\subsection{Stratifications}

Let $(A, \leqslant)$ be a poset, or simply $A$ when $\leqslant$ is clear from context.

Example 1 The set of simplices of a simplicial complex $C$ forms a poset under inclusion. This is called the face poset of $C$.

Remark 1 A poset $(A, \leqslant)$ may be interpreted as a category, whose objects are $A$ and $\operatorname{Hom}(a, b)=*$ if $a \leqslant b$ and $\emptyset$ otherwise. A poset may also be interpreted as a topological space endowed with the Alexandrov topology, whose basis contains open sets of the form $U_{a}:=\{b \in A: a \leqslant b\}$, for all $a \in A$.

Let $X$ be a topological space. Our definitions follow [13, Appendix A.5] and [4, Section 2].

Definition 2 An A-stratification of $X$ is a continuous map $f: X \rightarrow A$.

When $A$ is clear from context, $f$ is simply called a stratification, and $X$ is called $A$ stratified by $f$, or just $A$-stratified, or even stratified. We write $X_{a}:=\{x \in X: f(x)=a\}$ for the strata of $X$ and $A_{>a}:=\{b \in A: b>a\}$ for the subposet based at a particular element $a \in A$.

Given two stratifications $f: X \rightarrow A$ and $g: Y \rightarrow B$, a stratified map from $f$ to $g$ is a pair of continuous maps $\phi_{0}: X \rightarrow Y$ and $\phi_{1}: A \rightarrow B$ such that $g \circ \phi_{0}=\phi_{1} \circ f$. Such a stratified map is a homeomorphism, embedding, etc. whenever $\phi_{0}$ and $\phi_{0} \mid X_{a}$ have the same adjective, for every $a \in A$.

Definition 3 Let $X$ be a topological space. The open cone of $X$ is $C(X)=(X \times[0,1)) \cup\{*\}$, with $U \subseteq C(X)$ open whenever

- $\quad U \cap(X \times[0,1))$ is open, and

- $\quad X \times(0, \varepsilon) \subseteq U$ for some $\varepsilon>0$, if $* \in U$.

If $X$ is compact and Hausdorff, $C(X)=X \times[0,1) /(X \times\{0\})$.

When $X$ is $(A, \leqslant)$-stratified by $f, C(X)$ is naturally $\left(A^{\prime}, \leqslant\right)$-stratified by an induced map $f^{\prime}$, where $A^{\prime}:=A \cup\{\bullet\}$ and $\bullet \leqslant a$ for all $a \in A$. The stratifying map $f^{\prime}: C(X) \rightarrow A^{\prime}$ is given by $f^{\prime}(x, t)=f(x)$ for all $t \in(0,1)$, and $f^{\prime}(*)=\bullet$.

Definition 4 Let $f: X \rightarrow A$ be an $A$-stratification of $X$. Then $X$ is conically stratified at $x \in X$ by $f$ if there exist

- a topological space $Z$,

- $\quad$ an $A_{>f(x)}$-stratified topological space $L$, and

- $\quad$ an stratified map $Z \times C(L) \hookrightarrow X$ that is an open embedding whose image contains $x$.

The space $X$ is conically stratified by $f$ if it is conically stratified at every $x \in X$ by $f$, in which case we call $f$ a conical stratification of $X$.

The product $Z \times C(L)$ is canonically stratified by projection to the cone factor, that is, by the map $(z, c) \mapsto g(c)$ for $g$ a stratification of $C(L)$. 
Example 2 In Fig. 1, the spaces $C_{2}, S_{2}, S_{3}$ are conically stratified, while $C_{1}, C_{3}, S_{1}$ are not. The spaces $C_{1}, S_{1}$ fail to be conically stratified at every point on the equator, while $C_{3}$ fails to be conically stratified at the complex number 1 (see Example 3 ).

\section{Supporting Results}

In this section we develop ideas that support the main statements of Section 4. First we explore the implications for conical stratifications.

\subsection{Conical Stratifications}

An $A$-stratification of $X$ satisfies the frontier condition if $\left(\overline{X_{a}} \backslash X_{a}\right) \cap X_{b} \neq \emptyset$ implies $X_{b} \subseteq \overline{X_{a}}$, for every $a, b \in A$. See Fig. 1 for examples of spaces satisfying the frontier condition.

Lemma 1 Let $f$ be an A-stratification of a topological space $X$ whose strata are pathconnected. If $f$ is a conical stratification, then $f$ satisfies the frontier condition.

Proof Let $a, b \in A$. Since $X$ is conically stratified at $x \in X_{b}$, there is a stratified open embedding $\varphi: Z \times C(L) \rightarrow X$, as in Definition 4, for some $A_{>b}$-stratified space $L$.

First note that $L$ does not depend on $x$, as the image of $\varphi$ contains an open neighborhood $U_{x} \subseteq X$ of $x$, hence every element in $U_{x} \cap X_{b}=\varphi(Z \times *)$ has the same associated $L$ (up to a stratified homeomorphism). Indeed, suppose that $x^{\prime} \in X_{b}$ exists with an open embedding $\varphi_{x^{\prime}}: Z_{x^{\prime}} \times C\left(L_{x^{\prime}}\right)$ and $L \neq L_{x^{\prime}}$. Given a path $\gamma: I \rightarrow X_{b}$ from $x$ to $x^{\prime}$, letting $L_{\gamma(t)}$ be the $A_{>f(\gamma(t))}$-stratified space guaranteed to exist by Definition 4 , at $t^{\prime}=\sup _{t \in I}\left\{L_{\gamma(s)}=\right.$ $L \forall s \leqslant t\}$ we will arrive at a contradiction to the previous observation.

Next, suppose that $\left(\overline{X_{a}} \backslash X_{a}\right) \cap X_{b} \neq \emptyset$, and let $x \in\left(\overline{X_{a}} \backslash X_{a}\right) \cap X_{b}$.

Given the stratified cone $g: C(L) \rightarrow A \geqslant b$ from the embedding $\varphi$, it follows that $b \leqslant a$, since every open neighborhood of $x$ in $X$ intersects $X_{a}$. Hence $C(L)_{b} \subseteq \overline{C(L)_{a}}$, as the stratum $C(L)_{b}$ of the cone point $b$ is adjacent to all other strata of the cone, and $a$ is in the
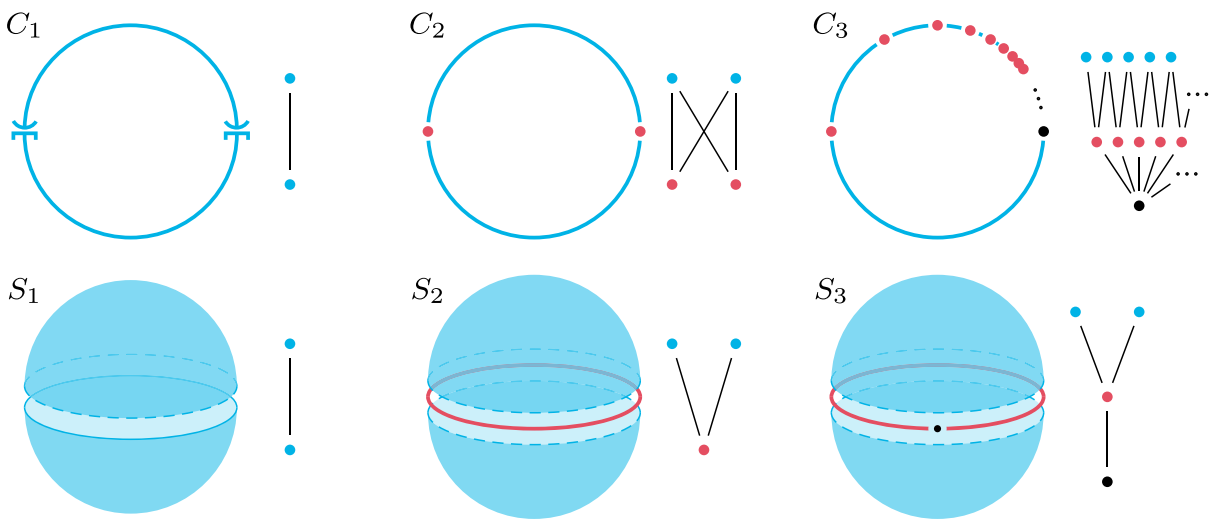

Fig. 1 Three stratifications of the circle and the sphere, with higher vertical position indicating higher order in the poset. The spaces $C_{1}$ and $C_{2}$ are great circles through the poles of $S_{1}$ and $S_{2}$, respectively. See Examples $2,3,4$ for observations about these stratifications 
image of $g$ by assumption. Hence $Z \times C(L)_{b} \subseteq \overline{Z \times C(L)_{a}}$, both viewed as subsets of $Z \times C(L)$. By continuity of the embedding $\varphi$, it follows that

$$
\varphi\left(Z \times C(L)_{b}\right) \subseteq \varphi\left(\overline{Z \times C(L)_{b}}\right) \subseteq \overline{\varphi\left(Z \times C(L)_{a}\right)} .
$$

Since $\varphi\left(Z \times C(L)_{b}\right) \subseteq X_{b}$, Eq. 4 means that $x$ has an open neighborhood $U_{x} \subseteq X_{b}$ such that $U_{x} \subseteq \overline{X_{a}}$.

Finally, since $L$ is the same for all elements of $X_{b}, a$ must be in the image of the associated cone map, and this is enough to conclude that every element of $X_{b}$ has a neighborhood within the closure of $X_{a}$. Hence $X_{b} \subseteq \overline{X_{a}}$.

The converse of Lemma 1 is false, as Example 3 shows.

Example 3 Consider the circle $C_{3}$ from Fig. 1, embedded as the unit circle in the complex numbers $\mathbf{C}$. This circle is stratified by the poset $(A, \leqslant)$, where $A=\left\{x_{1}, x_{2}, \ldots\right\} \cup$ $\left\{y_{1}, y_{2}, \ldots\right\}$, with relations $x_{j} \leqslant y_{j}$ and $x_{j+1} \leqslant y_{j}$ for all $j \in \mathbf{Z}_{>0}$. To ensure continuity of the stratifying map at the complex number 1 , we add the relations $x_{1} \leqslant x_{j}$ for all $j \in \mathbf{Z}_{\geqslant 2}$. The stratifying map $f: C_{3} \rightarrow A$ is given by

$$
f\left(e^{i \theta}\right)= \begin{cases}x_{j} & \text { if } \theta=2 \pi / j \\ y_{j} & \text { if } \theta \in(2 \pi /(j+1), 2 \pi / j) .\end{cases}
$$

That is, the black dot in $C_{3}$ in Fig. 1 corresponds to $x_{1}$, each red dot corresponds to an $x_{j} \geqslant 2$, and each blue interval corresponds to a $y_{j}$.

The frontier condition is satisfied trivially for strata $\left(C_{3}\right)_{x_{j}}$, as they are already closed in $C_{3}$. For $\left(C_{3}\right)_{y_{j}}$, note the closure of the open $\operatorname{arc}\left(C_{3}\right)_{y_{j}}=\left\{e^{i \theta}: \theta \in(2 \pi /(j+1), 2 \pi / j)\right\}$ contains exactly $\left(C_{3}\right)_{x_{j}}=\left\{e^{i 2 \pi / j}\right\}$ and $\left(C_{3}\right)_{x_{j+1}}=\left\{e^{i 2 \pi /(j+1)}\right\}$, hence the frontier condition is also satisfied here.

However, $C_{3}$ is not conically stratified at $1=e^{i 2 \pi}$. Indeed, following Definition 4 , we note that $Z$ must be $\{*\}$, as $\{1\}=\left(C_{3}\right)_{x_{1}}$ is 0 -dimensional. So if $C_{3}$ were conically stratified at 1 , there must be some open neighborhood of 1 that is the homeomorphic image of a cone $C(L)=Z \times C(L)$. To have an open embedding $C(L) \hookrightarrow C_{3}$, the cone $C(L)$ must have strata that correspond to strata in the open neighborhood of 1 . Since every neighborhood of 1 contains elements of the form $e^{i \theta}$ where $\theta \in(0, \varepsilon)$, for every $\varepsilon>0$, such a construction would imply that there are distinct 0 -dimensional strata in $C(L)$ corresponding to $\left(C_{3}\right)_{x_{\ell}}=$ $\left\{e^{i 2 \pi / \ell}\right\}$, for every integer $\ell>2 \pi / \varepsilon$. This is a contradiction, as the only 0 -dimensional stratum in $C(L)$ is the cone point.

An $A$-stratification of $X$ is compatible with, or refines a $B$-stratification of $X$ if for every $a \in A$ and $b \in B$, either $X_{a} \subseteq X_{b}$ or $X_{a} \cap X_{b}=\emptyset$. Equivalently, if for every $b \in B$ there is a subset $A^{\prime} \subseteq A$ such that $X_{b}=\bigcup_{a \in A^{\prime}} X_{a}$.

Example 4 In Fig. $1, C_{3}$ is compatible with $C_{2}$, and $C_{2}$ is compatible with $C_{1}$. Similarly, $S_{3}$ is compatible with $S_{2}$, and $S_{2}$ is compatible with $S_{1}$.

A stratification is semialgebraic if all its strata are semialgebraic sets.

Lemma 2 Let $f$ be a semialgebraic stratification of a closed semialgebraic set $X$. Then there exists a conical semialgebraic stratification of $X$ compatible with $f$. 
Proof Let $f: X \rightarrow A$ be as in the statement. By [15, Theorem II.4.2], there exists a simplicial complex $K$ whose geometric realization $|K|$ is homeomorphic to $X$, and a stratification $g:|K| \rightarrow(S(K), \subseteq)$ that refines $f$. We recall briefly that the geometric realization $|K|$ is a topological space embedded in Euclidean space, with $n$-simplices represented by $n$-dimensional subspaces.

This stratification of the geometric realization of a simplicial complex [13, Definition A.6.7] is the canonical one, identifying interiors of simplices with their corresponding simplices in the face poset $(S(K), \subseteq)$. This map is conical by [13, Proposition A.6.8].

The simplicial complex $K$ is unique (up to simplicial complex isomorphism) only if $X$ is bounded [15, Remark II.4.3]. Next we develop a new structure on simplicial complexes.

\subsection{Simplicial Complexes}

For $C, C^{\prime} \in \mathrm{SC}$, a simplicial map is a function $V(C) \rightarrow V\left(C^{\prime}\right)$ such that the induced map on $S(C)$ has image in $S\left(C^{\prime}\right)$. In other words, a simplicial map sends simplices of $C$ to simplices of $C^{\prime}$.

For $C \in \mathrm{SC}$, we denote by $[C]$ the set of simplicial complexes isomorphic to $C$. In a similar fashion, we write [SC] for the set of isomorphism classes of simplicial complexes.

Definition 5 Let $\succcurlyeq$ be the relation on [SC] given by $[C] \succcurlyeq\left[C^{\prime}\right]$ whenever there is a simplicial map $C \rightarrow C^{\prime}$ that is surjective on vertices.

Figure 2 gives an example of $\succcurlyeq$, with order decreasing from left to right. This relation is well-defined, irrespective of the choice of class representatives.

Lemma 3 The relation $\succcurlyeq$ defines a partial order on [SC].

Proof Let $[C],\left[C^{\prime}\right],\left[C^{\prime \prime}\right] \in[\mathrm{SC}]$. For reflexivity, take any two representatives $C_{1}, C_{2}$ of $[C]$. Since $C_{1} \cong C_{2}$, there is a bijection $C_{1} \rightarrow C_{2}$ in SC, which is surjective on vertices.

For anti-symmetry, suppose that $[C] \succcurlyeq\left[C^{\prime}\right]$ and $\left[C^{\prime}\right] \succcurlyeq[C]$. If $|V(C)|>\left|V\left(C^{\prime}\right)\right|$, then we cannot have $\left[C^{\prime}\right] \succcurlyeq[C]$, and if $\left|V\left(C^{\prime}\right)\right|>|V(C)|$, we cannot have $[C] \succcurlyeq\left[C^{\prime}\right]$. Hence we must have $|V(C)|=\left|V\left(C^{\prime}\right)\right|$, and so any map $C \rightarrow C^{\prime}$ inducing $[C] \succcurlyeq\left[C^{\prime}\right]$ must be injective on vertices, and so injective on simplices. Similarly, the same properties hold any map $C^{\prime} \rightarrow C$ inducing $\left[C^{\prime}\right] \succcurlyeq[C]$. Hence we have a map $C \rightarrow C^{\prime}$ that is bijective on simplices, so $C \cong C^{\prime}$, and $[C]=\left[C^{\prime}\right]$.

For transitivity, suppose that $[C] \succcurlyeq\left[C^{\prime}\right]$ and $\left[C^{\prime}\right] \succcurlyeq\left[C^{\prime \prime}\right]$. Then there exists a simplicial map $C \rightarrow C^{\prime}$ that is surjective on $V\left(C^{\prime}\right)$, as well a simplicial map $C^{\prime} \rightarrow C^{\prime \prime}$ that is surjective on $V\left(C^{\prime \prime}\right)$. The composition of these two simplicial maps is a simplicial map $C \rightarrow C^{\prime \prime}$, and

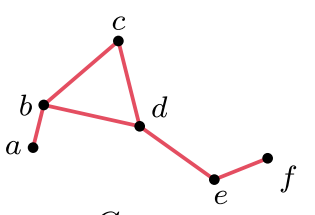

$C$

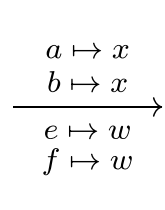

w

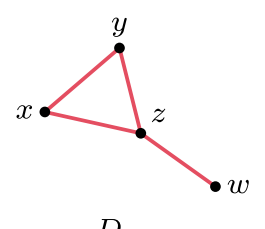

$D$

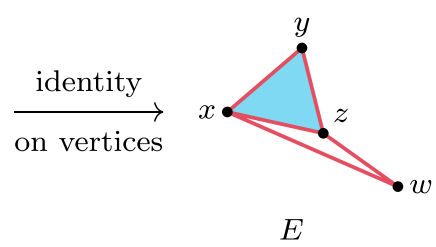

$E$

Fig. 2 An example of the relations $C \succcurlyeq D$ and $D \succcurlyeq E$ 
as both were individually surjective on vertices, the composition must also be surjective on vertices.

The same arguments show that $\succcurlyeq$ defines a preorder on SC. Figure 3 shows the Hasse diagram of ([SC], $\succcurlyeq)$ for all simplicial complexes up to 3 vertices.

Remark 2 The assumption that all simplicial complexes in SC are finite is key to proving Lemma 3, as anti-symmetry needs to compare sizes of sets. Figure 4 gives one such example where anti-symmetry is violated in the non-finite case.

\section{Main Results}

There is a natural point-counting map $\operatorname{Ran}(M) \rightarrow \mathbf{Z}_{>0}$, which is a stratification by [13, Remark 5.5.1.10], and is conical on $\operatorname{Ran}_{\leqslant n}(M)$ by [4, Proposition 3.7.5]. The goal of this section is to generalize the $\mathbf{Z}_{>0}$-stratification of $\operatorname{Ran}(M)$ to an [SC]-stratification of $\operatorname{Ran}(M) \times \mathbf{R}_{20}$.

\subsection{Stratifying $\operatorname{Ran}(\boldsymbol{M}) \times \mathbf{R}_{\geqslant 0}$}

We consider the partially ordered set ([SC], $\succcurlyeq)$ as a topological space with the Alexandrov topology. Let $[\check{C}]: \operatorname{Ran}(M) \times \mathbf{R}_{\geqslant 0} \rightarrow[\mathrm{SC}]$ be the composition of $\breve{C}$ and the projection to [SC].

Theorem 1 The $\check{C} e c h$ map $[\check{C}]$ is continuous.

Proof A basis for the Alexandrov topology on [SC] consists of the sets $U_{[C]}=\left\{\left[C^{\prime}\right] \in\right.$ [SC] : $\left.\left[C^{\prime}\right] \succcurlyeq[C]\right\}$ based at $[C] \in[\mathrm{SC}]$, so we show the preimage of any such set is open in $\operatorname{Ran}(M) \times \mathbf{R}_{\geqslant 0}$. Take any $(P, r) \in[\breve{C}]^{-1}\left(U_{[C]}\right)$, with $P=\left\{P_{1}, \ldots, P_{k}\right\}$, which we will show has an open neighborhood contained in $[\check{C}]^{-1}\left(U_{[C]}\right)$. For every $P^{\prime} \subseteq P$, let

$$
\begin{aligned}
\check{c} s\left(P^{\prime}\right) & :=\bigcap_{p \in P^{\prime}} \bar{B}\left(p, \inf \left\{r: \bigcap_{p^{\prime} \in P^{\prime}} \bar{B}\left(p^{\prime}, r\right) \neq \emptyset\right\}\right) \subseteq M, \\
\check{c} r\left(P^{\prime}, r\right) & :=r-d_{M}\left(P^{\prime}, \check{c} s\left(P^{\prime}\right)\right) \in \mathbf{R}
\end{aligned}
$$

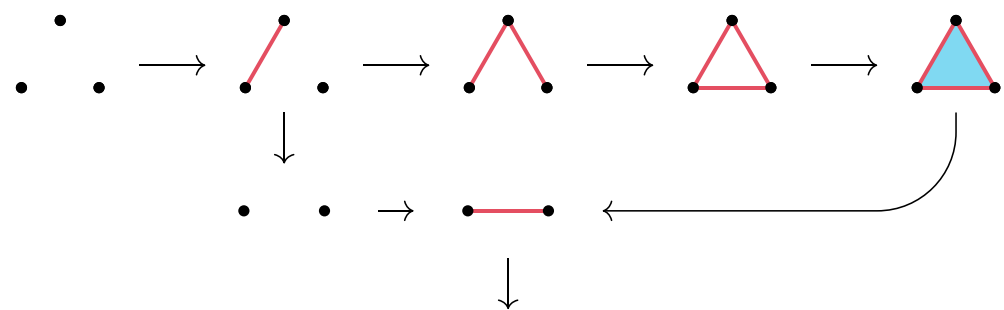

Fig. 3 Relations in the poset ([SC], $\succcurlyeq)$, with arrows indicating simplicial maps and decreasing partial order. Horizontal simplicial maps are injective, vertical maps are not. Compare with Fig. 7 


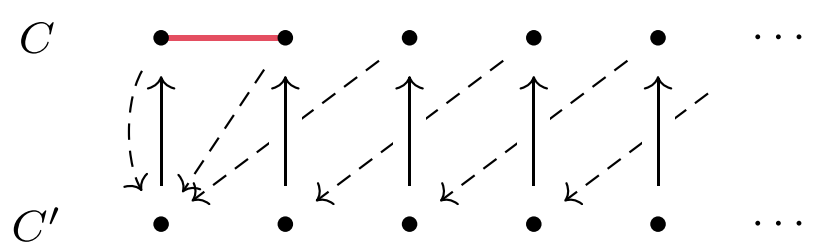

Fig. 4 Two infinite simplicial complexes $C, C^{\prime}$ with maps $C^{\prime} \rightarrow C$ (solid lines) and $C \rightarrow C^{\prime}$ (dashed lines) described on vertices. Both maps are surjective on vertices, but the simplicial complexes are not isomorphic

be the $\check{C} e c h s e t^{3}$ of $P^{\prime}$ and $\check{C} e c h$ radius of $P^{\prime}$ at $r$, respectively. ${ }^{4}$ The $\check{C}$ ech set is the smallest non-empty intersection of the closed balls on $M$ of increasing radius around $P^{\prime}$. The inf is necessary when $\left|P^{\prime}\right|=1$, otherwise the minimum always exists, as the balls are closed and $M$ is connected. The Čech radius is positive if and only if the intersection $\bigcap_{p \in P^{\prime}} \bar{B}(p, r)$ contains an open set of $M$, negative when the intersection is empty, and 0 otherwise.

Case 1: For every $P^{\prime} \subseteq P$ with $\left|P^{\prime}\right|>1, \check{c} r\left(P^{\prime}, r\right) \neq 0$. Let $B_{\infty}((P, r), \tilde{r} / 4)$ be the open ball in the sup-norm on the product $\operatorname{Ran}(M) \times \mathbf{R} \geqslant 0$ around $(P, r)$ of radius $\tilde{r} / 4$, where $\tilde{r}$ is the smallest of the two values

$$
\begin{aligned}
& r_{1}:=\min _{1 \leqslant i<j \leqslant k} d\left(P_{i}, P_{j}\right), \\
& r_{2}:=\min _{P^{\prime} \subseteq P,\left|P^{\prime}\right|>1} 2\left|\breve{c} r\left(P^{\prime}, r\right)\right| .
\end{aligned}
$$

Briefly, having $\tilde{r} \leqslant r_{1}$ guarantees that points will not merge in the open ball, and having $\tilde{r} \leqslant r_{2}$ guarantees that simplices among the $P_{i}$ are neither lost nor gained in the open ball. Figure 5 illustrates these roles of $r_{1}$ and $r_{2}$.

Let $(Q, s) \in B_{\infty}((P, r), \tilde{r} / 4)$. Since $\tilde{r} \leqslant r_{1}$, we have that $d_{H}(P, Q)<\tilde{r} / 4$, which implies that $Q \subseteq \bigcup_{i=1}^{k} B\left(P_{i}, \tilde{r} / 4\right)$. Similarly, the $B\left(P_{i}, \tilde{r} / 4\right)$ are disjoint. Also, for every $1 \leqslant i \leqslant k$, note that $Q \cap B\left(P_{i}, \tilde{r} / 4\right) \neq \emptyset$, as

$$
d_{M}\left(\left\{P_{i}\right\}, Q\right)=\min _{q \in Q} d\left(P_{i}, q\right) \leqslant d_{H}(P, Q) \leqslant d_{\infty}((P, r),(Q, s))<\tilde{r} / 4 .
$$

In other words, there is a well-defined and surjective map $\phi: Q \rightarrow P$ for which $\phi(q)=P_{i}$ whenever $q \in B\left(P_{i}, \tilde{r} / 4\right)$.

Next, we claim $\phi$ is a simplicial map. Take $Q^{\prime} \subseteq Q$ and suppose that $\check{C}\left(Q^{\prime}, s\right)$ is a $\left(\left|Q^{\prime}\right|-1\right)$-simplex. Let $P^{\prime}=\left\{P_{0}^{\prime}, \ldots, P_{\ell}^{\prime}\right\} \subseteq P$ be such that $Q^{\prime} \subseteq \bigcup_{i=1}^{\ell} B\left(P_{i}^{\prime}, \tilde{r} / 4\right)$ and $Q \cap B\left(P_{i}^{\prime}, \tilde{r} / 4\right) \neq \emptyset$, for $1 \leqslant i \leqslant \ell$. Suppose, for contradiction, that $\check{C}\left(P^{\prime}, r\right)$ is not a $\left(\left|P^{\prime}\right|-1\right)$-simplex, or equivalently, that $\breve{c} r\left(P^{\prime}, r\right)<0$. Then

$$
\begin{array}{rlr}
0 & \geqslant \check{c} r\left(P^{\prime}, r\right)+\tilde{r} / 2 & \text { (by Eq. } \left.8 \text { and that } \tilde{r} \leqslant r_{2}\right) \\
& =r-d_{M}\left(P^{\prime}, \check{c} s\left(P^{\prime}\right)\right)+\tilde{r} / 2 & \text { (by definition of Čech radius) } \\
& >r-d_{M}\left(Q^{\prime}, \check{c} s\left(Q^{\prime}\right)\right)-\tilde{r} / 4+\tilde{r} / 2 & \left(\text { since } d_{H}(P, Q)<\tilde{r} / 4\right) \\
& \geqslant s-|s-r|-d_{M}\left(Q^{\prime}, \check{c} s\left(Q^{\prime}\right)\right)+\tilde{r} / 4 & \\
& >\check{c} r\left(Q^{\prime}, s\right)-\tilde{r} / 4+\tilde{r} / 4 & \\
& =\check{c} r\left(Q^{\prime}, s\right),
\end{array}
$$

\footnotetext{
${ }^{3}$ This can be thought of as the circumcenter of some subset of $P$, whose size is restricted by $\operatorname{dim}(M)$ and whose choice is restricted by its convex hull.

${ }^{4}$ These two constructions are related by the equation $\check{c} r\left(P^{\prime}, d_{M}\left(P^{\prime}, \check{c} s\left(P^{\prime}\right)\right)\right)=0$.
} 


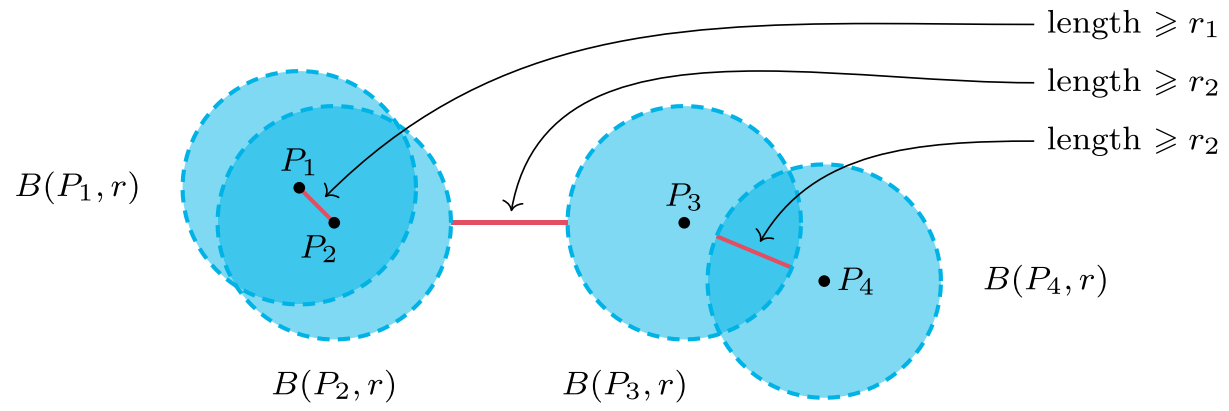

Fig. 5 A finite subset of $M$ and open balls in $M$ around its elements

contradicting the assumption that $\check{c} r\left(Q^{\prime}, s\right) \geqslant 0$, as $\check{C}\left(Q^{\prime}, s\right)$ was assumed to be a $\left(\left|Q^{\prime}\right|-1\right)$ simplex. Hence $\check{C}\left(P^{\prime}, r\right)$ is a $\left(\left|P^{\prime}\right|-1\right)$-simplex, and so the image of $\check{C}\left(Q^{\prime}, s\right)$ under $\phi$ is the simplex $\check{C}\left(P^{\prime}, r\right)$. Since simplices get taken to simplices, the map $\phi: Q \rightarrow P$ extends to a simplicial map $\check{C}(Q, s) \rightarrow \breve{C}(P, r)$ that is surjective on vertices. That is, $[\check{C}(Q, s)] \succcurlyeq$ $[\check{C}(P, r)]=[C]$, and so $B_{\infty}((P, r), \tilde{r} / 4) \subseteq[\check{C}]^{-1}\left(U_{[C]}\right)$, meaning that $[\check{C}]^{-1}\left(U_{[C]}\right)$ is open.

Case 2: There is some $P^{\prime} \subseteq P$ with $\left|P^{\prime}\right|>1$ and $\check{c} r\left(P^{\prime}, r\right)=0$. Then $r_{2}=0$ from Eq. 8, so let

$$
r_{2}^{\prime}:=\min _{P^{\prime} \subseteq P, \breve{c} r\left(P^{\prime}, r\right) \neq 0} 2\left|\check{c} r\left(P^{\prime}, r\right)\right|,
$$

and let $\tilde{r}$ be the smallest of the two values $r_{1}$ and $r_{2}^{\prime}$. As in Case 1, we claim the open neighborhood $B_{\infty}((P, r), \tilde{r} / 4)$ of $(P, r)$ is contained within $[\check{C}]^{-1}\left(U_{[C]}\right)$. The proof of this claim proceeds as in the first case: the only place that $r_{2}$ was used was to state that $0 \geqslant$ $\check{c} r\left(P^{\prime}, r\right)+\tilde{r} / 2$, in showing that $\check{C}\left(P^{\prime}, r\right)$ is indeed a $\left(\left|P^{\prime}\right|-1\right)$-simplex. If $\check{c} r\left(P^{\prime}, r\right)=0$, then we already have this conclusion, and it is unnecessary to get to the contradiction. That is, $\phi$ still extends to a simplicial map, and $[\check{C}]^{-1}\left(U_{[C]}\right)$ is open in this case as well.

It follows that $[\check{C}]$ is a [SC]-stratification of $\operatorname{Ran}(M) \times \mathbf{R}_{\geqslant 0}$. Moreover, $[\check{C}]$ is a refinement of the point-counting stratification $\operatorname{Ran}(M) \times \mathbf{Z}_{>0}$, by viewing $\mathbf{Z}_{>0}$ as a subposet of discrete simplicial complexes of $[\mathrm{SC}]$ by the map $n \mapsto(\{1, \ldots, n\},\{\{1\}, \ldots,\{n\}\})$.

However, Lemma 1 implies that $[\check{C}]$ is not a conical stratification.

Example 5 Consider the space of at most 2 points $\operatorname{Ran}_{\leqslant 2}(I)$ on the unit interval $I$, and the space $X=\operatorname{Ran}_{\leqslant 2}(I) \times \mathbf{R}_{\geqslant 0}$, as shown in Fig. 6 . Take $x=\left(\left\{p_{1}, p_{2}\right\}, r\right) \in X$, with $p_{1}=0, p_{2}=\frac{1}{2}$ and $r=\frac{1}{4}$. For $y=\left(\left\{p_{1}, p_{2}\right\}, r^{\prime}<r\right)$, note that

$$
a=\bullet \cdot=[\check{C}](y) \succcurlyeq[\check{C}](x)=\bullet=b .
$$

Moreover, $y$ is in the closure of of both $X_{b}$ and $X_{a}$, that is, $\left(\overline{X_{a}} \backslash X_{a}\right) \cap X_{b} \neq \emptyset$. However, for $z=\left(\left\{p_{1}, p_{2}\right\}, r^{\prime \prime}>r\right)$ we see that $z \in X_{b}$ and $z \notin X_{a}$, meaning that $X_{b} \nsubseteq \overline{X_{a}}$. Hence $[\check{C}]$ does not satisfy the frontier condition, and so by Lemma 1 cannot be a conical stratification.

One solution is to make a new stratum for points similar to $x$ in Example 5. That is, for every $[C] \in[\mathrm{SC}]$, declare a new stratum $S_{[C]}=\left\{(P, r) \in \operatorname{Ran}(M) \times \mathbf{R}_{\geqslant 0}:[\check{C}](P, r)=\right.$ $[C], \check{c} r(P, r)=0\}$. 

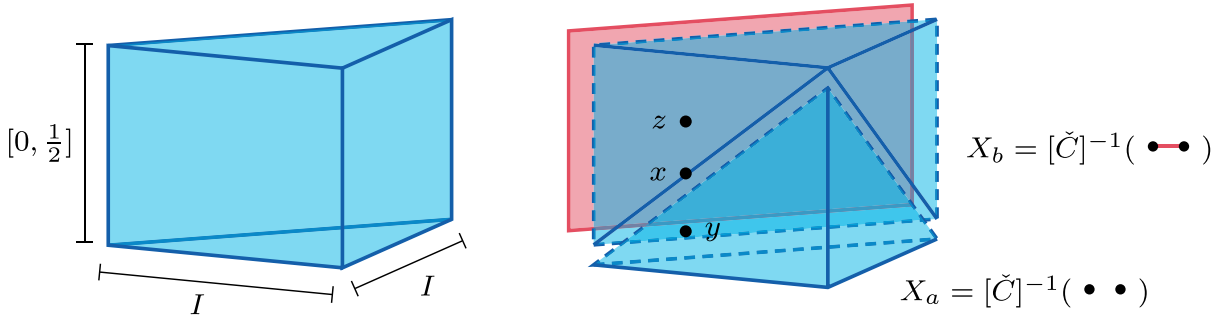

Fig. 6 The stratified space $\operatorname{Ran}_{\leqslant 2}(I) \times \mathbf{R}_{\geqslant 0}$ decomposed into its strata (right)

Definition 6 A frontier simplicial complex $C$ is defined by its vertices, simplices, and frontier simplices, that is, a triple of sets $(V(C), S(C), F(C))$ with $(V(C), S(C))$ a simplicial complex and $F(C) \subseteq S(C)$ closed under taking supersets.

By "closed under taking supersets" we mean $\sigma \in F(C)$ implies $\tau \in F(C)$ whenever $\sigma \subseteq \tau$ and $\tau \in S(C)$. A map of frontier simplicial complexes $(V, S, F) \rightarrow\left(V^{\prime}, S^{\prime}, F^{\prime}\right)$ is defined analogously to a map of simplicial complexes. That is, we require it to be a map on the vertices $V \rightarrow V^{\prime}$ which must induce a map on simplices $S \rightarrow S^{\prime}$ and on frontier simplices $F \rightarrow F^{\prime}$. Figure 7 shows maps among all non-empty frontier simplicial complexes with at most 3 vertices.

Given a pair $(P, r) \in \operatorname{Ran}(M) \times \mathbf{R}_{\geqslant 0}$, augmenting $\check{C}(P, r)$ with the set $F$ such that $P^{\prime} \in F$ whenever $P^{\prime} \in S(\check{C}(P, r))$ and $\check{c} r\left(P^{\prime}, r\right)=0$ defines a frontier simplicial complex. This follows as the Čech radius is 0 when the intersection of closed $r$-balls around the elements of $P$ is non-empty but does not contain an open set.

Remark 3 Let FSC be the set of frontier simplicial complexes, for which we say $(V, S, F)=C \cong C^{\prime}=\left(V^{\prime}, S^{\prime}, F^{\prime}\right)$ whenever $(V, S) \cong\left(V^{\prime}, S^{\prime}\right)$, and the isomorphism on vertices induces an isomorphism $F \cong F^{\prime}$. It follows that:

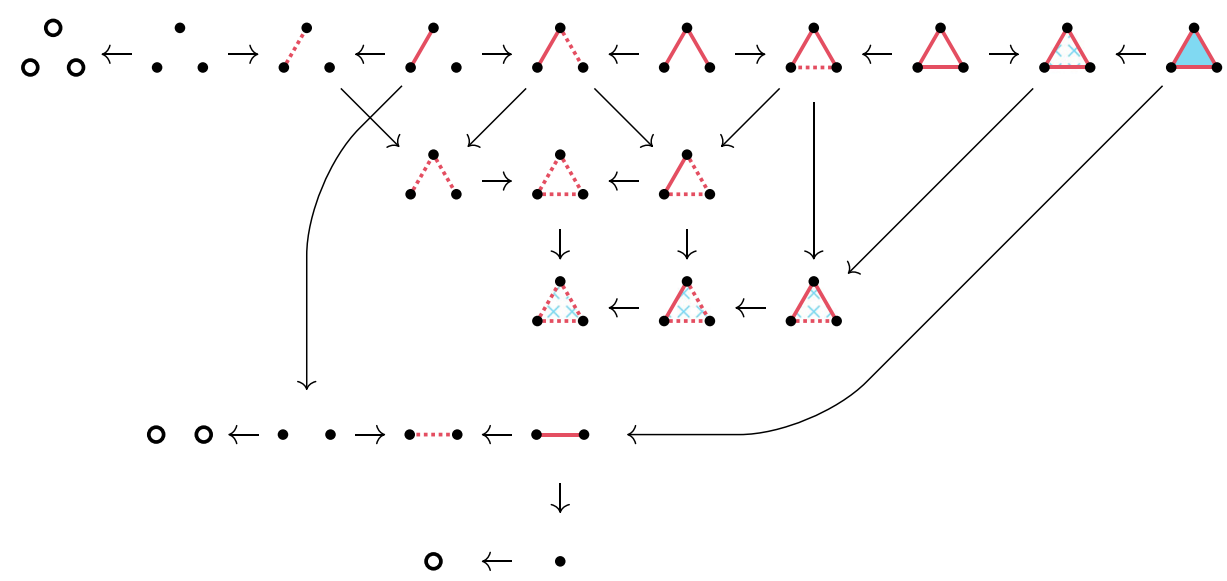

Fig. 7 Enrichment of Fig. 3 by frontier simplices, with arrows indicating simplicial maps and decreasing partial order in [FSC]. Frontier simplices are drawn as circles, dotted edges, hatched faces. Frontier simplicial complexes not in the image of the Čech map to [FSC] from Observation 3 are not shown 

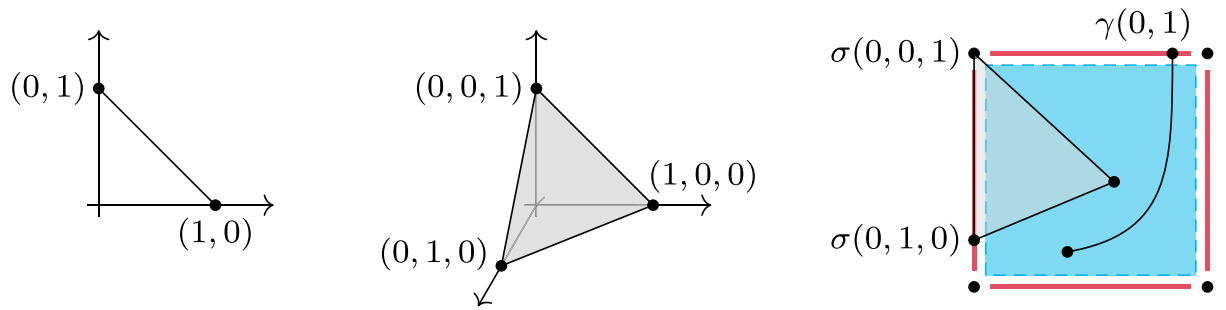

Fig. 8 Geometric realizations $\left|\Delta^{1}\right|$ (left), $\left|\Delta^{2}\right|$ (center), and their images as entrance paths $\gamma, \sigma$, respectively, in a stratified square (right)

- $\quad$ The Čech map factors as $\operatorname{Ran}(M) \times \mathbf{R}_{\geqslant 0} \rightarrow$ FSC $\rightarrow$ SC, first following the construction above, then forgetting frontier simplices.

- The set $[\mathrm{FSC}]:=\mathrm{FSC} / \cong$ has a partial order by letting $[C] \succcurlyeq\left[C^{\prime}\right]$ whenever there is a simplicial map $C \rightarrow C^{\prime}$ that is surjective on vertices and injective on frontier simplices.

- The induced map $\operatorname{Ran}(M) \times \mathbf{R}_{\geqslant 0} \rightarrow[\mathrm{FSC}]$ is continuous.

The last statement follows as the proof of Theorem 1 was split up into two cases where the Čech radius is and is not zero, so all that remains is to keep track of the frontier simplices throughout the proof.

Conjecture 1 The induced map $\operatorname{Ran}(M) \times \mathbf{R}_{\geqslant 0} \rightarrow[\mathrm{FSC}]$ is a conical stratification.

Remark 4 We mention two observations to support Conjecture 1.

- This stratification does not immediately violate the frontier condition on pathconnected components of strata like $[\check{C}]$ does.

- Each 1-dimensional frontier simplex of $C \in$ [FSC] decreases the dimension of an open neighborhood in the stratum of $C$, relative to $C \in[\mathrm{SC}]$.

The second statement implies Example 5 cannot be immediately used with this stratification. However, the statement only seems to hold up to some relationship between $|V(C)|$ and the dimension of an open neighborhood of $V(C) \subseteq M$.

For a clearer result, we restrict to semialgebraic sets and fix an upper bound $n \in \mathbf{Z}_{>0}$. We also employ some results about the algebra of semialgebraic sets, specifically that products [15, I.2.9.1], quotients [9, Corollary 1.5], sub-semialgebraic sets [8, Theorem 9.1.6], and images via semialgebraic maps [15, I.2.9.11] are all semialgebraic.

The function $[\check{C}]$ now refers to the restriction of $[\check{C}]$ to $\operatorname{Ran}_{\leqslant n}(M) \times \mathbf{R}_{\geqslant 0}$.

Theorem 2 If $M$ is semialgebraic, there exists a conical semialgebraic stratification of $\operatorname{Ran}_{\leqslant n}(M) \times \mathbf{R}_{\geqslant 0}$ compatible with $[\check{C}]$.

Proof Since $M$ is semialgebraic, [15, I.2.9.1] gives that $M^{n}$ is semialgebraic. By describing $\operatorname{Ran}_{\leqslant n}(M)$ as a quotient of $M^{n}$ by semialgebraic equivalence relations, [9, Corollary 1.5] gives that $\operatorname{Ran}_{\leqslant n}(M)$ is semialgebraic. Again by [15, I.2.9.1] we get that $\operatorname{Ran}_{\leqslant n}(M) \times \mathbf{R}_{\geqslant 0}$ is semialgebraic.

Now we show the strata are semialgebraic sets. Consider the set $[\check{C}]^{-1}([C]) \subseteq$ $\operatorname{Ran}_{\leqslant n}(M) \times \mathbf{R}_{\geqslant 0}$, which is defined by functions which use the distance from a point $(P, r)$ 
to its $\check{C}$ ech set $\check{c} s(P)$. The Čech set, from Eq. 5, is a semialgebraic set, as it is the intersection of balls, and the function that measures distance to a semialgebraic set is also semialgebraic, by [15, I.2.9.11]. Finally, a subset of a semialgebraic set defined by semialgebraic functions on the first set is itself semialgebraic in $\mathbf{R}^{N}$, by [8, Theorem 9.1.6]. Hence $[\check{C}]^{-1}([C])$ is semialgebraic, so $[\check{C}]$ is a semialgebraic stratification of $\operatorname{Ran}_{\leqslant n}(M) \times \mathbf{R} \geqslant 0$. Apply Lemma 2 to get a conical semialgebraic stratification of $\operatorname{Ran}_{\leqslant n}(M) \times \mathbf{R}_{\geqslant 0}$ compatible with $[\check{C}]$.

\subsection{Stratifying Paths}

For $X$, a topological space, recall $\operatorname{Sing}(X)$ is the simplicial set of continuous maps $\left|\Delta^{k}\right| \rightarrow$ $X$, where $\Delta^{k}$ is the standard $k$-simplex. Let $A$ be a poset and $f: X \rightarrow A$ a stratification.

Definition 7 An entrance path in $X$ is a continuous map $\sigma:\left|\Delta^{k}\right| \rightarrow X$ for which there exists a chain $a_{0} \leqslant \cdots \leqslant a_{k}$ in $A$ such that $f\left(\sigma\left(0, \ldots, 0, t_{i}, \ldots, t_{k}\right)\right)=a_{k-i}$ and $t_{i} \neq 0$, for all $i$.

Contrast this with the more common definition of an exit path, as in [18], which is the same, but with $f\left(\sigma\left(t_{0}, \ldots, t_{i}, 0, \ldots, 0\right)\right)=a_{i}$ and $t_{i} \neq 0$, for all $i$. The choice of "entrance" instead of "entry" comes from interpreting "exit" as a noun rather than a verb. Some examples of entrance paths are given in Fig. 8.

The subsimplicial set of $\operatorname{Sing}(X)$ of all entrance paths is denoted $\operatorname{Sing}_{A}(X)$. In this context, a very roundabout way of defining the Čech map $\check{C}$ from Definition 1 would be as an assignment

$$
\text { Čech }_{0}: \operatorname{Sing}_{[\mathrm{SC}]}\left(\operatorname{Ran}(M) \times \mathbf{R}_{\geqslant 0}\right)_{0} \rightarrow N(\mathrm{SC} c a t)_{0}
$$

of 0 -simplices, where $N(-)$ is the nerve. This description is useful, however, when generalizing from points ( 0 -simplices) to paths (1-simplices), in which case we only have to change the subscripts from 0 to 1 .

Construction 1 For an entrance path $\gamma:\left|\Delta^{1}\right| \rightarrow \operatorname{Ran}(M) \times \mathbf{R}_{\geqslant 0}$, we have

$$
[\check{C}](\gamma(0,1))=C, \quad[\check{C}](\gamma(t, 1-t))=C^{\prime} \forall t \in(0,1],
$$

and $C^{\prime} \succcurlyeq C$. Since $[\check{C}](\gamma(t, 1-t))$ is constant for all $t \in(0,1]$, the image of $\gamma$ is in $\operatorname{Conf}_{k}(M) \times \mathbf{R}_{\geqslant 0}$ for all $t \in(0,1]$ and $k=\left|V\left(C^{\prime}\right)\right|$. That is, there are paths $\gamma_{i}:\left|\Delta^{1}\right| \rightarrow M$ for $i=1, \ldots, k$, unique up to reindexing, such that the diagram

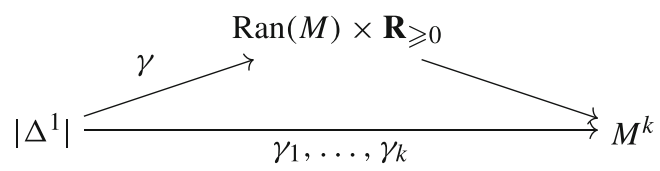

commutes. The $\gamma_{i}$ define a map from the vertices of $\check{C}(\gamma(1,0))$ to $\check{C}(\gamma(0,1))$, which in turn defines a simplicial map from $\check{C}(\gamma(1,0))$ to $\check{C}(\gamma(0,1))$ that is surjective on vertices. Call this simplicial map

$$
\check{C} \operatorname{ech}_{1}(\gamma) \in \operatorname{Hom}_{S C} c a t(\check{C}(\gamma(1,0)), \check{C}(\gamma(0,1))) .
$$

Note that two different $\gamma, \gamma^{\prime}$ maps with the same endpoints may not induce the same simplicial map Čech $1(\gamma)$, Čech $1\left(\gamma^{\prime}\right)$. That is, monodromy information is lost in the associated simplicial map, as demonstrated in Fig. 9.

Remark 5 Recall from Section 3.2 that a simplicial map $C \rightarrow C^{\prime}$ is a map $V(C) \rightarrow$ $V\left(C^{\prime}\right)$ which, when applied to elements of $S(C)$, gives elements of $S\left(C^{\prime}\right)$. The claim in 


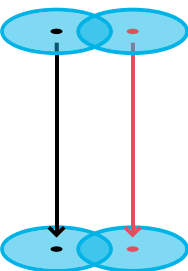

$\gamma_{1}$

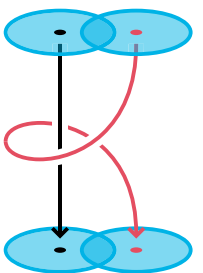

$\gamma_{2}$

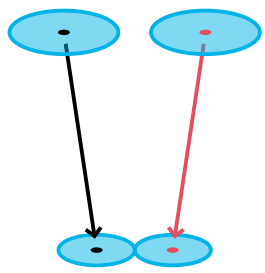

$\gamma_{3}$

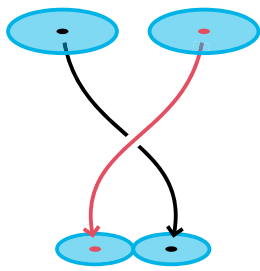

$\gamma_{3}$

Fig. 9 The class $[\check{C}]\left(\gamma_{i}(t, 1-t)\right)$ for $i=1,2$ is constant for all $t$, and for $i=3,4$ is constant only for $t \in(0,1]$. The simplicial maps associated to $\gamma_{1}$ and $\gamma_{2}$ are both the identity, while the map associated to $\gamma_{3}$ is different from the one associated to $\gamma_{4}$ (and neither are the identity)

Construction 1 that the $\gamma_{i}$ satisfy the conditions of a simplicial map follows by several observations:

- Any $\gamma_{i}(t, 1-t)$ may coincide only for $t=0$, that is, at the end of the path.

- An intersection $\bigcap_{i} \bar{B}\left(\gamma_{i}(t, 1-t), r_{t}\right)$ that is non-empty for $t=1$ must be non-empty for all $t \in(0,1]$, else $\gamma$ would not be an entrance path.

- The only possibility of $\bigcap_{i} \bar{B}\left(\gamma_{i}(t, 1-t), r_{t}\right)$ being non-empty for all $t \in(0,1]$ and empty for $t=0$ is if $r_{0}=0$, in which case all the $\gamma_{i}(0,1)$ coincide, which describes a surjective map from a simplex to a single vertex.

- Since the balls $\bar{B}$ are closed, it is impossible to preserve simplicial complex isomorphism class by making one intersection empty at the same instant $t \in(0,1)$ as another is made non-empty.

Here we have used $r_{t}$ for the $\mathbf{R}_{\geqslant 0}$ component of $\gamma(t, 1-t)$.

Considering $\check{C}$ as $\check{C ̌ e c h}_{0}$ and with the construction above of $\check{C ̌ e c h}_{1}$, we are tempted to generalize the result further.

Conjecture 2 Čech $_{0}$ and Čech ${ }_{1}$ extend to a map Čech: $\operatorname{Sing}_{[\mathrm{SC}]}\left(\operatorname{Ran}(M) \times \mathbf{R}_{\geqslant 0}\right) \rightarrow$ $N$ (SCcat) of simplicial sets.

Examples abound of $C, C^{\prime} \in \mathrm{SC}$ with different simplicial maps $C \rightarrow C^{\prime}$ that are surjective on vertices, but it is not immediate that it is possible to construct an entrance path into some [SC]-stratified $\operatorname{Ran}(M) \times \mathbf{R}_{\geqslant 0}$ joining such simplicial maps. That is, we do not immediately find counterexamples to Conjecture 2, so we hope it is true.

We conclude this section with some observations about paths.

Remark 6 Let $\gamma:\left|\Delta^{1}\right| \rightarrow \operatorname{Ran}(M) \times \mathbf{R}_{\geqslant 0}$ be a path and $\check{\gamma}=[\check{C}] \circ \gamma$.

1. The subposet $\operatorname{im}(\check{\gamma}) \subseteq[\mathrm{SC}]$ corresponds to a zigzag of simplicial complexes and simplicial maps.

2. If $\gamma$ is contained in $\operatorname{Conf}_{n}(M)$ and $\operatorname{im}(\check{\gamma})$ is totally ordered by $\succcurlyeq$, then $\operatorname{im}(\check{\gamma})$ is a filtration of a simplicial complex on $n$ points.

3. If $\gamma(1-t, t)=(P, t /(1-t))$, then $\check{\gamma}$ corresponds to the Čech filtration of $P \subseteq M$.

These observations describe $\operatorname{Ran}(M) \times \mathbf{R} \geqslant 0$ as a topological space of simplicial complex filtrations, as illustrated in Fig. 10. 


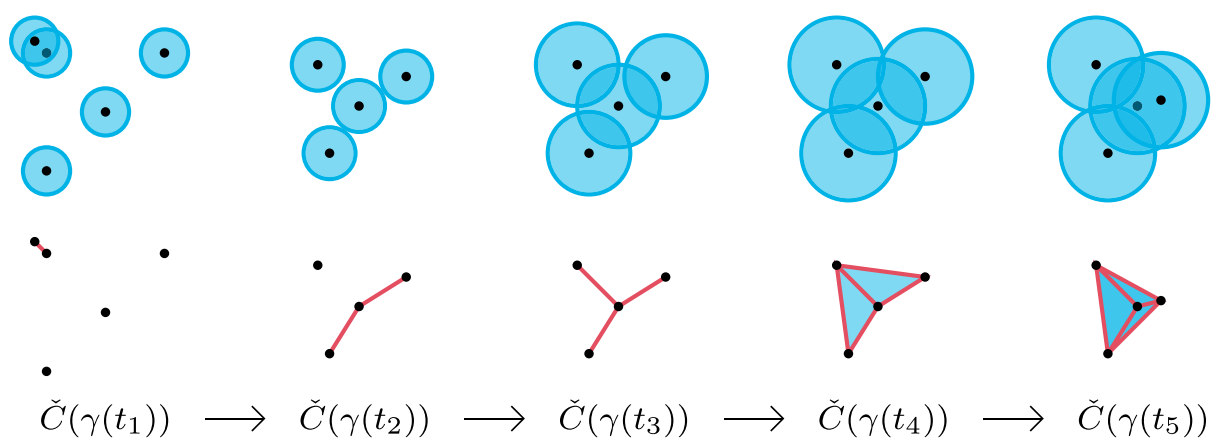

Fig. 10 A path in $\operatorname{Ran}(M) \times \mathbf{R}_{\geqslant 0}$ and its corresponding zigzag in SC. Restricting to [ $\left.t_{2}, t_{4}\right]$ we have part of the Čech filtration on the $\operatorname{Ran}(M)$ component of $\gamma\left(t_{2}\right)$

\section{Discussion}

We have presented a thorough description of the space $\operatorname{Ran}(M) \times \mathbf{R}_{\geqslant 0}$, motivated by its interpretation as the space of all simplicial complexes on a metric space $M$. Our description gives a stratification $[\check{C}$ ] based on the Čech construction of a simplicial complex on $M$. This stratification may be refined into a structurally cleaner but more opaque conical stratification (Theorem 2), as well as a combinatorially motivated stratification, though it is unclear if the latter is conical (Remark 3 ). We use $[\check{C}]$ to associate paths with simplicial maps in Section 4.2, relating them to existing constructions in persistent homology (Remark 6) and conjecturing that the association extends to continuous maps of higher-dimensional simplices (Conjecture 2).

This approach prompts questions about the new concepts we introduced:

- What does the poset of frontier simplicial complexes look like?

- Is the [FSC]-stratification of $\operatorname{Ran}(M) \times \mathbf{R}_{\geqslant 0}$ conical?

We are also motivated to push further the inquiry into interpreting paths:

- Does the Čech map and its generalization Čech 1 to paths extend to higher-dimensional simplices?

The choice of working with isomorphism classes of simplicial complexes, in which the vertices have no order, and simplicial sets (for the entrance paths and the nerve), in which the 0 -simplices are ordered, does not make our work easier. An alternative approach would have been to take the nerve of the face poset of a simplicial complex, which is a simplicial set, instead of the simplicial complex itself. Part of the appeal of using isomorphism classes is that less information is remembered, hence it is not immediate that using simplicial sets would help.

Acknowledgements The author was partly sponsored by EP/P025072/1. Thanks to Ben Antieau and Shmuel Weinberger for insightful discussions and guidance. Thanks to Justin Curry for suggesting the counterexample of Fig. 4. Thanks to the reviewers for helpful comments and corrections.

Data Availability Data sharing not applicable to this article as no datasets were generated or analysed during the current study. 
Open Access This article is licensed under a Creative Commons Attribution 4.0 International License, which permits use, sharing, adaptation, distribution and reproduction in any medium or format, as long as you give appropriate credit to the original author(s) and the source, provide a link to the Creative Commons licence, and indicate if changes were made. The images or other third party material in this article are included in the article's Creative Commons licence, unless indicated otherwise in a credit line to the material. If material is not included in the article's Creative Commons licence and your intended use is not permitted by statutory regulation or exceeds the permitted use, you will need to obtain permission directly from the copyright holder. To view a copy of this licence, visit http://creativecommons.org/licenses/by/4.0/.

\section{References}

1. Adamaszek, M., Adams, H., Frick, F.: Metric reconstruction via optimal transport. SIAM J. Appl. Algebra Geom. 2(4), 597-619 (2018). https://doi.org/10.1137/17M1148025

2. Adams, H., Carlsson, G.: Evasion paths in mobile sensor networks. Int. J. Robot. Res. 34(1), 90-104 (2015). https://doi.org/10.1177/0278364914548051

3. Ayala, D., Francis, J., Rozenblyum, N.: A stratified homotopy hypothesis. J. Eur. Math. Soc. (JEMS) 21(4), 1071-1178 (2019). https://doi.org/10.4171/JEMS/856

4. Ayala, D., Francis, J., Tanaka, H.L.: Local structures on stratified spaces. Adv. Math. 307, 903-1028 (2017). https://doi.org/10.1016/j.aim.2016.11.032

5. Bauer, U., Lesnick, M.: Induced matchings and the algebraic stability of persistence barcodes. J. Comput. Geom. 6(2), 162-191 (2015)

6. Beilinson, A., Drinfeld, V.: Chiral Algebras, American Mathematical Society Colloquium Publications, vol. 51. American Mathematical Society, Providence (2004). https://doi.org/10.1090/coll/051

7. Blumberg, A.J., Lesnick, M.: Universality of the homotopy interleaving distance. ArXiv e-prints (2017)

8. Bochnak, J., Coste, M., Roy, M.F.: Real Algebraic Geometry, Ergebnisse Der Mathematik Und Ihrer Grenzgebiete (3) [Results in Mathematics and Related Areas (3)], vol. 36. Springer, Berlin (1998). https://doi.org/10.1007/978-3-662-03718-8. Translated from the 1987 French original, Revised by the authors

9. Brumfiel, G.W.: Quotient spaces for semialgebraic equivalence relations. Math. Z. 195(1), 69-78 (1987). https://doi.org/10.1007/BF01161599

10. Bubenik, P., Vergili, T.: Topological spaces of persistence modules and their properties. J. Appl. Comput. Topol. 2(3-4), 233-269 (2018). https://doi.org/10.1007/s41468-018-0022-4

11. Carlsson, G.: Topology and data. Bull. Amer. Math. Soc. (N.S.) 46(2), 255-308 (2009). https://doi.org/10.1090/S0273-0979-09-01249-X

12. Knudsen, B.: Configuration spaces in algebraic topology. arXiv:1803.11165 (2018)

13. Lurie, J.: Higher algebra. http://www.math.harvard.edu/lurie/papers/HA.pdf (2017)

14. Niyogi, P., Smale, S., Weinberger, S.: Finding the homology of submanifolds with high confidence from random samples. Discrete Comput. Geom. 39(1-3), 419-441 (2008). https://doi.org/10.1007/s00454008-9053-2

15. Shiota, M.: Geometry of Subanalytic and Semialgebraic Sets, Progress in Mathematics, vol. 150. Birkhäuser Boston, Inc., Boston (1997). https://doi.org/10.1007/978-1-4612-2008-4

16. Singh, G., Memoli, F., Carlsson, G.: Topological methods for the analysis of high dimensional data sets and 3D object recognition. In: Botsch, M., Pajarola, R., Chen, B., Zwicker, M. (eds.) Eurographics symposium on point-based graphics. The Eurographics Association (2007). https://doi.org/10.2312/SPBG/ SPBG07/091-100

17. Topaz, C.M., Ziegelmeier, L., Halverson, T.: Topological data analysis of biological aggregation models. PLOS ONE 10(5), 1-26 (2015). https://doi.org/10.1371/journal.pone.0126383

18. Treumann, D.: Exit paths and constructible stacks. Compos. Math. 145(6), 1504-1532 (2009). https://doi.org/10.1112/S0010437X09004229

19. Weinberger, S.: The Topological Classification of Stratified Spaces. Chicago Lectures in Mathematics. University of Chicago Press, Chicago (1994)

20. Whitney, H.: Local properties of analytic varieties. In: Differential and combinatorial topology (A Symposium in Honor of Marston Morse), pp. 205-244. Princeton Univ. Press, Princeton (1965)

21. Zomorodian, A., Carlsson, G.: Computing persistent homology. Discrete Comput. Geom. 33(2), 249274 (2005). https://doi.org/10.1007/s00454-004-1146-y

Publisher's Note Springer Nature remains neutral with regard to jurisdictional claims in published maps and institutional affiliations. 